\title{
RESPONSABILIDAD DEL CONTADOR PÚBLICO EN LA EVALUACIÓN CONTINUA DE LAS TIC EN EMPRESAS CON CONTABILIDAD ON-LINE
}

\author{
RESPONSIBILITY ACCOUNTING FROM THE ONGOING EVALUATION OF \\ ICT IN COMPANIES WITH ONLINE ACCOUNTING
}

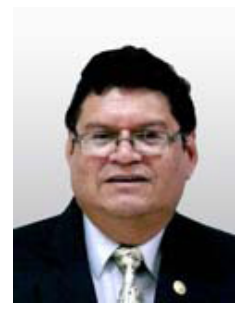

\section{Carlos Alberto Pastor Carrasco *}

Docente Asociado de la Facultad de Ciencias Contables- UNMSM

[Recepción: Setiembre de 2011 / Conformidad: Octubre de 2011]

\section{Resumen}

Los avances tecnológicos (por ejemplo, el comercio electrónico e Internet) han cambiado las prácticas de negocios y el proceso de registro y almacenamiento de las transacciones comerciales. Extensible Business Reporting Language (XBRL) pronto será incorporado en el software de contabilidad y presentación de informes que permitan on-line en tiempo real la preparación, publicación, examen, y la extracción de la información financiera. Por lo tanto, los auditores externos, independientes deberán utilizar la evaluación continua, cuando la mayoría de la información financiera que exista en las organizaciones sea sólo en forma electrónica en los sistemas de contabilidad y en tiempo real. La Evaluación continua y sus implicaciones para los auditores independientes, son incluidas en el presente documento. Asimismo son descritas y analizadas las consideraciones de control interno y procedimientos de auditoría, En el futuro, serán comunes las auditorías sin papeles como ocurre a los clientes de auditoría que cada vez más cambian a sistemas sin papel. El software de auditoría permite a los auditores completar la mayoría de los procedimientos en línea. Para auditar los sistemas en línea, los auditores tendrán que incorporar un software en línea de auditoría como herramienta de auditoría primaria y reunir pruebas por medios electrónicos. Debe evaluarse el impacto de la tecnología en el proceso de auditoría, y se analizan las implicaciones futuras de las tendencias tecnológicas para la profesión auditora. Más específicamente, se ofrece un resumen de cómo la tecnología de la información ha afectado la planificación de auditorías, pruebas y documentación.

Palabras Claves: Tiempo real, sistema de contabilidad, Evaluación de tecnología de la información, sistemas de información, Internet, Auditoria.

\begin{abstract}
Technological advances (eg, electronic commerce and the Internet) have changed business practices and the process of recording and storing business transactions. Extensible Business Reporting Language (XBRL) will soon be incorporated into the accounting software and reporting that allow on-line real-time preparation, publication, examination, and extraction of financial information. Therefore, external auditors, independent evaluation should be used continuously, when most financial information exists in organizations is only in electronic form in the accounting systems and in real time. Continuous Assessment and its implications for independent auditors, are included in this document. They are also described and analyzed internal control considerations and audit procedures, Future audits will be common as is paperless audit clients increasingly shift to paperless systems. The developed audit software allows auditors to complete most procedures on-line. For online systems audit, auditors will need to incorporate an online software audit as a primary audit tool and gather evidence electronically. Should assess the impact of technology on the audit process, and discusses the future implications of technological trends for the auditing profession. More specifically, it provides an overview of how information technology has impacted audit planning, testing and documentation.
\end{abstract}

Keywords: Real-time accounting system, Evaluation of information technology, information systems, Internet, Audit

* Magister en Ingeniería de Sistemas e Informática- UNMSM- Docente Asociado de la Facultad de Ciencias Contables UNMSM. Contador Público Colegiado Certificado. Ha presentado trabajos de su especialidad en Conferencias Interamericanas de Contabilidad en Brasil y Bolivia. E-mail: cpc5637@hotmail.com 


\section{INTRODUCCIÓN}

La emergente Tecnología de la información y comunicación (TIC) ha dado lugar a nuevos enfoques de negocios: como el comercio electrónico, el intercambio electrónico de datos (EDI) y finalmente Internet. Estos nuevos enfoques han cambiado totalmente las prácticas de negocios y el proceso de registro y almacenamiento de las transacciones comerciales y sus correspondientes informes financieros y contables. En estos momentos, hacer negocios en la Web a través del uso de la tecnología de Internet permite a las organizaciones conectarse con el mundo en línea y les permite mejorar todos los aspectos de su negocio. El sitio Web se ha convertido en un elemento que puede mejorar la venta de productos o servicios, dando opciones a los compradores, actuales o potenciales, a comprar productos o servicios directamente en línea.

En la actualidad, la mayor parte de las transacciones comerciales pueden ser efectuadas en forma electrónica, sin contar con ningún tipo de documentación en papel, permitiendo a las organizaciones presentar información financiera en tiempo real, en línea. Es así, que las empresas van a pasar de un sistema de intercambio electrónico de Datos (EDI), relativamente costoso a otras opciones menos costosas y más flexibles tales como el Extensible Markup Language (XML). Además, en la actualidad se está desarrollando el Extensible Markup Language de Información Financiera (XFRML) para facilitar a las empresas en el intercambio de información financiera. (Pastor, 2009)

Estas nuevas Tecnologías de información (ejemplo: Internet, EDI, XML, XFRML) han cruzado las fronteras nacionales para cambiar la forma de comunicación en nuestras organizaciones. Muchas entidades ya divulgan sus informes trimestrales y anuales a través de Internet. Extensible Business Reporting Language (XBRL) está recibiendo el apoyo y la popularidad de la comunidad financiera y la profesión contable. XBRL es un lenguaje estandarizado electrónico para la presentación de negocios que facilita la preparación, publicación, examen, y la extracción de la información financiera.

En XBRL, la información se introduce sólo una vez y luego se puede representar en cualquier forma, como una declaración impresa financieras, un documento HTML para el sitio Web de la empresa, y documento de archivo EDGAR de la SEC, o cualquier otro formato de presentación de informes especializados (Zarowin y Harding, 2000). Fomenta la preparación efectiva y eficaz, el intercambio automático y la extracción confiable de información financiera a través de todos los formatos de tecnología, incluida la Internet.

XBRL reduce repetir procesos de información financiera y proporciona una plataforma para los sistemas en línea en tiempo real la contabilidad. Los auditores deben utilizar la evaluación continua de las TIC, cuando la mayoría de la información financiera que se procesa en la entidad sólo sea en forma electrónica.

Los propósitos principales de este trabajo son los siguientes:

1. Poner en discusión el Tema de la Evaluación continua de las TIC (ECTIC) y sus implicaciones para la profesión contable;

2. Examinar el control interno de las TIC, las cuales se encuentran en constante cambio, y

3. Finalmente, examinar los aspectos clave de la auditoría de las nuevas TIC's. 


\section{EVALUACIÓN CONTINUA DE LA TECNOLOGÍA DE INFORMACIÓN Y COMUNICACIONES}

La toma de decisiones de alta calidad y en forma oportuna, dependen en gran parte de la calidad de los datos y la existencia de información en línea y en tiempo real. La información electrónica y digital es más flexible, accesible, transferible, y puede ser más fácil de almacenar, resumir, y organizar que la información en papel. Las Tecnologías de la información han permitido a las organizaciones llevar a cabo sus transacciones comerciales por vía electrónica y preparar sus estados financieros en un sistema en línea y en tiempo real. En sistemas con Contabilidad en Tiempo Real (Contabilidad on-line), tanto la información financiera y la evidencia de auditoría van a estar disponibles sólo en formato electrónico. Los documentos fuentes de uso tradicional como: las órdenes de compra, facturas y cheques son reemplazados por mensajes electrónicos, y los datos subyacentes de contabilidad (por ejemplo, revistas, libros y calendarios) se encuentran en los formularios electrónicos o archivos (Rezaee et al., 2000).

El cambio en el proceso de negocio mediante el cual se elimina una fuente tradicional de información (Libros y registros en papel), requiere la creación de nuevos procedimientos de auditoría para llevar a cabo la auditoría financiera. Debemos tener presente que el principal objetivo de la auditoría financiera y las normas de auditoría generalmente aceptadas (NAGA) no cambian porque toda o una parte de los registros de los clientes sea realizada en formato electrónico.

Sin embargo, lo que si puede variar son los procedimientos de auditoría, cuando la mayoría de la información sólo existe en forma electrónica en el sistema de Contabilidad on-line, Lo que obliga al Contador auditor a emplear la Evaluación Continua de Tecnología de Información y Comunicación (ECTIC). ECTIC puede definirse como "proceso sistemático de recolección de evidencia de auditoría electrónica como una base razonable para emitir una opinión sobre la presentación razonable de estados financieros, preparados sin el uso de papeles o en medios electrónicos en tiempo real". ECTIC, en otras palabras, es un proceso de recopilación y evaluación de las pruebas para determinar la eficiencia y la eficacia de la Contabilidad on-line, soportada en medios electrónicos que permite salvaguardar los activos, el mantenimiento de la integridad de datos, y producir información financiera confiable.

La realización de una Evaluación de Tecnología de Información Continua conlleva emplear una serie de tecnologías emergentes de auditoría, tales como: utilización de software automatizado, técnicas de ETIC, módulos integrados de auditoría, servicios integrados de prueba, y herramientas de auditoría concurrente.

Dichas técnicas de auditoría concurrente permiten a los auditores diseñar e implementar códigos de programas que serán empleados directamente a una gran variedad de aplicaciones de computadora para seleccionar y supervisar continuamente el tratamiento de los datos.

Luego de la investigación realizada por el suscrito, en algunas empresas de Lima, se llegó a las siguientes tres conclusiones principales:

1. Las evaluaciones continuas de las TIC son viables, siempre que ciertas condiciones, se cumplan;

2. Las Investigaciones de académicos, profesionales de la experimentación y la orientación de los organismos de nor- 
malización son necesarias para ayudar a los servicios de Evaluación continua de Tecnología de Información y comunicaciones; $y$,

3. Debido a la demanda de más confiabilidad, relevancia y oportuna toma de decisiones, es probable que se cree la necesidad de implementar una Evaluación Continua de Tecnología de Información y Comunicaciones, para lo cual el Contador Público necesita capacitarse para responder adecuadamente al mercado.

El proceso de ECTIC es la evolución de las auditorias manuales de los sistemas de contabilidad sustentadas con documentación en papel, a la auditoría electrónica continua del EDI, en la que los sistemas contables no están sustentadas en papel, sino en medios electrónicos.

Los auditores convencionales se están dando cuenta de que las auditorías manuales (en torno a la auditoría informática) no son eficientes en los sistemas de Contabilidad on-line. Una auditoría de estados financieros en una organización que tenga Contabilidad on-line, carece de los accesos a la documentación de referencia tradicional, dado que han sido eliminados o son almacenados en forma exclusivamente electrónica. Esta situación afecta al proceso de revisión en varias maneras.

En primer lugar, el conocimiento del auditor del negocio del cliente y la industria tiene que aumentar, para asegurar la fiabilidad y relevancia de los documentos electrónicos, registros y datos.

En segundo lugar, el auditor tiene que entender mejor el flujo de las transacciones y actividades efectuadas en forma electrónica que estén relacionadas con el control, para asegurar la validez y fiabilidad de la información en un entorno sin papel, (sistema Contabilidad on-line).

En tercer lugar, el auditor tiene que usar un control de riesgos orientando el plan de auditoría para que se centre principalmente en la adecuación y eficacia de las actividades de control inter- no del sistema de Contabilidad on-line y el lugar menos prominente en las pruebas sustantivas de los documentos y transacciones electrónicas. Hay muchas maneras diferentes de abordar y planificar la evaluación continua de Tecnología de Información en entidades con una Contabilidad on-line, así por ejemplo con el uso de programas de software de aplicación (por ejemplo, la extracción de datos y análisis interactivos (IDEA) y XBRL).

La principal ventaja de la utilización de Evaluación de Tecnología de Información Continua (ETIC), es la reducción del costo de llevar a cabo un trabajo de auditoría. ETIC permite a los auditores poner a prueba una muestra más grande (hasta 100 por ciento) de las transacciones de los clientes y los datos más rápidamente y más eficientemente que la prueba manual de auditoría de todo el equipo. ETIC puede reducir la cantidad de tiempo y costes tradicionalmente dedicado a la exploración manual de las transacciones y saldos de las cuentas. ETIC también puede aumentar la calidad de las auditorías financieras al permitir a los auditores centrarse más en la comprensión del negocio del cliente y de la industria y su estructura de control interno.

Las firmas de contabilidad se están alejando de las auditorías y trámites tradicionales mediante el programa de auditoría basado en Web y la ETIC. Por ejemplo, Deloitte \& Touche, en cooperación con Intacct Corp., están en proceso de desarrollo de la primera evaluación basada en la Web del programa de auditoría para pequeñas y medianas empresas (Intacct Corp., 2000). Los clientes que proporcionan información financiera continúan a lo largo del año, lo que permitiría ETIC, en lugar de auditar los libros de la compañía en el sitio al final del año.

A continuación se muestra la visión general del ciclo de contabilidad en los siste- 
mas on-line y el proceso de auditoría relacionados con la ETIC.

Un sistema de contabilidad en línea consta de los siguientes procesos:

1. Identificación de las transacciones y otros acontecimientos económicos;

2. Medición, reconocimiento y reporte de transacciones en on-line, sistema de información contable en tiempo real;

3. Existencia de una estructura de control interno adecuados y eficaces;

4. Procesamiento de las transacciones por vía electrónica;

5. Contabilidad General en línea, y

6. Preparación en línea y en tiempo real de los estados financieros.

La Evaluación continua de las TIC debe de constar de cinco fases:

1. Planificación de un trabajo de auditoría, incluidos los procedimientos analíticos;

2. Examen de la estructura de control interno de la Contabilidad on-line incluso la aplicación de pruebas de los controles y la evaluación del riesgo de control;

3. Ejecución de las pruebas sustantivas provisionales y permanente de los detalles de las transacciones;

4. Realizar las pruebas sustantivas de saldos de las cuentas y los resultados generales, incluidos los procedimientos de análisis, y

5. Completar la auditoría y la emisión de un informe de auditoría.

Durante la etapa de planificación, los auditores deben prestar atención a la disponibilidad y auditabilidad de los formularios electrónicos, registros y documentos. El examen de la estructura de control interno es similar a la de un sistema manual, que requiere que el auditor para comprender todos los cinco componentes de la estructura de control. Los auditores pueden reunir evidencia suficiente y competente por vía electrónica como una base razonable para emitir una opinión sobre los estados financieros.

\section{CONTROLES INTERNOS}

Los nuevos avances tecnológicos han aumentado la importancia en los controles internos.

Los cinco componentes de la estructura de control interno que indica en el informe COSO son: mecanismos de control, evaluación de riesgos, información y comunicación, las actividades de control y monitoreo. Una suficiente comprensión de estos cinco componentes de control permitirá ayudar a los auditores para decidir si un control es adecuado para las actividades en que se basan en un sistema contable on-line. Si existen procedimientos de control adecuados, entonces el auditor deberá realizar pruebas de controles para determinar la efectividad de las políticas internas de control de la estructura y procedimientos para prevenir, detectar y corregir errores materiales en los estados financieros.

La Evaluación del riesgo determina el grado de fiabilidad de la estructura de control interno. Un examen y revisión del sistema de control interno es importante en un sistema de Contabilidad on-line. Esta revisión de los controles internos de ayuda a los auditores independientes evaluar el riesgo del control interno y emitir un dictamen sobre el grado de fiabilidad que se puede colocar en la estructura de control interno de un sistema de contabilidad on-line.

La fiabilidad de los controles internos es la base para la determinación de la naturaleza, oportunidad y alcance de los procedimientos sustantivos de auditoría realizado en la recopilación de evidencia suficiente y competente.

Tradicionalmente, los auditores independientes realizan pruebas de control para evaluar la idoneidad y la eficacia de la estructura de control interno 
Los avances tecnológicos han aumentado la importancia en los controles internos (naturaleza, oportunidad y extensión) y por ello sus pruebas. Los auditores independientes realizan pruebas de controles para reunir evidencia que sirva de base para reducir las pruebas sustantivas más costosas.

Sin embargo, bajo el sistema de Contabilidad on-line, los auditores deben realizar pruebas de los controles al mismo tiempo que pruebas sustantivas de detalles de transacciones de reunir pruebas sobre la fiabilidad del sistema en la producción fiable y creíble de la información financiera. Las pruebas de los controles comienzan con la revisión del auditor y la comprensión de controles de gestión. $\mathrm{Si}$ estos controles no operan efectivamente como estaba previsto, a continuación, podría haber necesidad de probar los controles de aplicación. Si los auditores deciden que la gestión controles son adecuados y eficaces, que luego evaluar la suficiencia y eficacia de los aplicación de los controles relacionados con el material clases de transacciones en los diversos subsistemas de aplicación.

Un examen de los acuerdos comerciales incluye el examen y evaluación de los controles de gestión y de sistema de aplicación de los controles y evaluar el ambiente de control y componentes de evaluación de riesgos de los internos estructura de control mediante el examen de la gestión los controles (por ejemplo, comité de auditoría, estilo de funcionamiento, gestión de riesgo). Los auditores deben estudiar y evaluar el control específico de las actividades mediante el examen de gestión y el control de la aplicación del sistema (por ejemplo, de entrada, procesamiento, producción y control de gestión).

Las técnicas más utilizadas en las pruebas de auditoría asistida por ordenador de la eficacia de la estructura de control interno, entre otros, son los siguientes:
1. Los datos de prueba o prueba de instalaciones integradas en la determinación de si el sistema Contable on-line está procesando correctamente las transacciones válidas y no válidas y en la verificación de exactitud e integridad de procesamiento;

2. De simulación en paralelo en el desarrollo de un programa informático que reproduce una parte del sistema de solicitud de un cliente en la evaluación de la eficacia de las actividades de control;

3. De procesamiento concurrente en el diseño de módulos de auditoría y otros códigos de programación y ejecución de ellos directamente en las aplicaciones más importantes para seleccionar de forma continua y supervisar el tratamiento de los datos, $y$

4. De simulación continua e intermitente, que se utiliza para seleccionar las transacciones durante el proceso de revisión de auditoría y proporcionar una capacidad de auditoría on-line.

5. Técnicas de auditoría concurrente, tales como el enfoque instantáneo, el control de sistemas y la revisión de instalaciones se espera que reciban una mayor atención $y$ el uso de ECTIC pruebe su eficacia en la estructura de control interno del cliente.

6. Las pruebas obtenidas mediante la realización de pruebas de los controles proporciona una base para los auditores para evaluar el riesgo de control y para finalizar la auditoría el plan mediante la determinación de la naturaleza, oportunidad y el alcance de las pruebas sustantivas.

\section{ASPECTOS DE AUDITORIA DE TI}

Los auditores independientes deben considerar la disponibilidad de realizar pruebas en formato electrónico y sus implicaciones para determinar el alcance de las pruebas de los controles y la naturaleza, oportunidad y alcance de las pruebas sustantivas. Cada vez 
más el uso de TI y el comercio electrónico requiere que los auditores obtengan evidencia electrónica y, en consecuencia, debe fomentarse en la profesión contable a incorporar el concepto de la prueba electrónica en su nivel de calidad.

Las entidades que transmiten, procesan, mantienen o acceden a grandes cantidades de información electrónica pueden ser incapaces de reducir el riesgo de detección a un nivel aceptable, por tal motivo no es suficiente que los auditores desempeñen sólo procedimientos sustantivos, sino que deben realizar normalmente las pruebas de controles para obtener evidencia para ayudar a lograr un nivel de riesgo de control lo suficientemente por debajo del máximo. Ciertas pruebas electrónicas deben existir en un momento determinado, pero puede no estar disponible después de un período determinado, si los archivos se cambian y los archivos de copia de seguridad no existen. Motivo por el cual el auditor debe considerar un tiempo durante el cual la información existe o está disponible en la determinación de la naturaleza, oportunidad y alcance de las pruebas sustantivas.

Cada trabajo de auditoría implica la evaluación de las afirmaciones de la administración (por ejemplo, la existencia de los activos, pasivos y patrimonio neto, la calidad de las ganancias, la fiabilidad del control interno, cumplimiento de las leyes y reglamentos aplicables) al reunir evidencia suficiente y competente. Para grandes entidades muy computarizadas, la evidencia puede ser exclusivamente en forma electrónica, mientras que para las organizaciones pequeñas y tradicionales de la evidencia puede ser todavía en un formato en papel del documento. Por lo tanto, es apropiado adoptar diferentes procedimientos de auditoría y pruebas para cada uno de estos trabajos de auditoría.
Para emitir un informe de auditoría, el auditor deberá determinar:

1. ¿Qué pruebas se requieren para hacer frente a cada afirmación;

2. ¿Qué procedimientos de auditoría debe realizar para reunir evidencia competente y convincente para cada una de las afirmaciones;

3. Determinar cuánto es suficiente la evidencia obtenida; $y$,

4. Recolección de evidencia suficiente $y$ competente de medios más fiable y eficiente.

Evaluación continúa a las TIC d un sistema de Contabilidad on-line requiere que los auditores cuenten con evidencia suficiente y competente, para lo cual deben obtener respuestas convincentes a las preguntas siguientes:

1. ¿Se encuentran disponibles los registros electrónicos?

2. ¿Es política del cliente la retención de dichos registros?

3. ¿Qué actividades de control realizan para salvaguardar los registros?

4. ¿Se encuentran los detalles y las actas disponibles para el período de la auditoría?

5. ¿Son fiables los registros electrónicos?

6. ¿Se cuenta con controles de encriptación y autenticación para garantizar la integridad de los documentos electrónicos?

7. ¿Es la estructura de control interno adecuado y efectivo para garantizar la fiabilidad de las pruebas electrónicas?

8. ¿De dónde se obtienen los datos de los estados financieros (Ejem. aspectos financieros, inventario)?

9. ¿Cuáles son los orígenes de los registros electrónicos de los clientes?

10. ¿Existe un registro de auditoría y en qué medida se aplica?

11. ¿Cuándo, dónde y cómo serán auditados los registros y documentos electrónicos? 
12. ¿Se encuentra la evidencia de auditoría en la computadora del cliente para ser auditados en sus instalaciones?

13. ¿El auditor tiene el hardware y los recursos de software disponibles para llevar a cabo una auditoría de las pruebas electrónicas?

14. ¿Qué paquetes de software de auditoría están disponibles?

15. ¿Qué técnicas computarizadas están disponibles para simplificar la evidencia de auditoría electrónica?
Los Auditores deben aplicar procedimientos de auditoría para reunir evidencia competente y suficiente para satisfacer el estándar de la tercera parte de trabajo de campo y utilizarlo como una base razonable para expresar una opinión sobre la presentación razonable de los estados financieros.

Las pruebas sustantivas son procedimientos de auditoría diseñadas a prueba de errores. Hay tres tipos de pruebas sustantivas: los procedimientos analíticos, pruebas de detalles de transacciones y las pruebas de detalle de los saldos

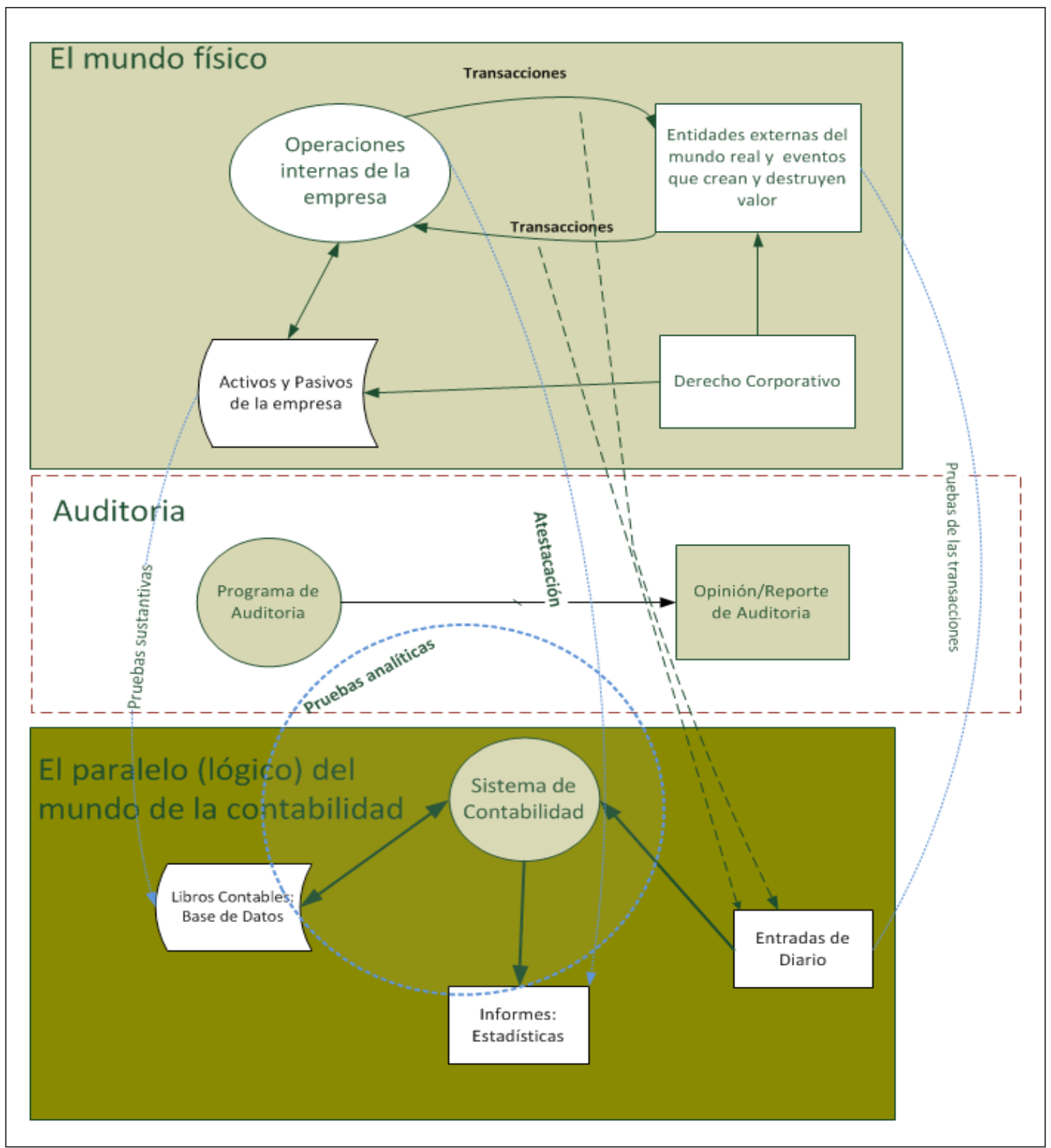

192/ QUIPURAMAYoc | Vol. 19(36) 2011 


\section{PRUEBAS ANALÍticaS}

Los procedimientos analíticos son los estudios de comparaciones y relaciones entre los datos financieros. Los procedimientos analíticos normalmente implican el cálculo de los coeficientes y su comparación con los de años anteriores. Por lo tanto, el uso de la ECTIC hace que sea más viable para realizar los procedimientos analíticos. Se requiere que los auditores utilicen los procedimientos analíticos en la planificación y última fase de presentación de informes de los trabajos de auditoría. Los procedimientos analíticos deben ser realizados durante la fase de planeación de la auditoría para entender mejor el negocio del cliente y la industria. También se puede utilizar durante la fase de recolección de pruebas de la auditoría para proporcionar una indicación de la presencia de errores posibles en los estados financieros y la posible reducción de la extensión de las pruebas de detalles de transacciones y saldos de las cuentas. Los procedimientos analíticos también se realizan durante la fase de elaboración del informe final de la auditoría para evaluar:

1. Resultados de auditoría en general;

2. Razonabilidad de las transacciones y saldos; y,

3. Capacidad de la entidad para continuar como negocio en marcha.

Con el uso de técnicas de auditoría concurrente y la tecnología informática, los procedimientos analíticos pueden ser menos costosas pruebas de auditoría para llevar a cabo.

\section{PRUEBAS DE LAS TRANSACCIONES}

Los auditores realizan pruebas de las transacciones para determinar si el tratamiento erróneo o irregular de operaciones ha causado errores significativos en los estados financieros. Estas pruebas incluyen rastreo de las entradas del diario de documentos de su origen o fuente de los documentos dando fe a sus entradas de diario relacionadas. Las técnicas de auditoría informática de ayuda son muy útiles en la realización de estas pruebas intermedias y la recopilación de pruebas para las pruebas finales de los saldos. En ECTIC, los auditores deben realizar pruebas de transacciones continuamente durante todo el año, en los periodos intermedios, a fin de reducir el alcance de las pruebas sustantivas de saldos que se realiza después de la fecha del balance. Si las pruebas reunidas por la realización de pruebas de las transacciones indica que los errores materiales y se han cometido irregularidades o si no lo hace o es que la información financiera o puede ser tergiversado materialmente, las pruebas de fondo de los saldos se ampliará. En virtud de ETIC, las pruebas de las transacciones se deben realizar coincidiendo con las pruebas de controles como las pruebas de doble propósito.

\section{PRUEBAS SUSTANTIVAS DE SALDOS}

En las pruebas sustantivas de saldos, con la ECTIC, se llevarán a cabo después de la fecha del balance para reunir pruebas suficientes competentes como una base razonable para expresar una opinión sobre la presentación razonable de los estados financieros.

Algunos ejemplos pueden ser: pruebas de confirmación de saldos de cuentas por cobrar, los recuentos físicos de inventario, y el nuevo cálculo de los pasivos de pensiones, o la depreciación de los activos a largo plazo.

Puede ser utilizado un Software de auditoría generalizado para realizar pruebas de confirmación de saldos, por ejemplo, seleccionar e imprimir las confirmaciones. Pruebas de detalle de los saldos se pueden realizar en los saldos finales de las partidas financieras de balance como de las cuentas de resultados. Sin embargo, se centran principalmente en las partidas del balance.

El alcance y la naturaleza de las pruebas de los saldos deben depender de los resultados de 
las pruebas de los controles, los procedimientos analíticos y pruebas sustantivas de las transacciones. Estas pruebas son eficaces, ya que a menudo incluyen el uso de documentación externa o el conocimiento personal directo del auditor, sino que son los más costosos y requerir mucho tiempo para llevar a cabo.

\section{CONCLUSIONES}

1. Un sistema de contabilidad en tiempo real permite a las organizaciones mantener sus informes financieros, listas de clientes, catálogos de piezas y listas de precios actualizadas, en línea, y de fácil acceso tanto a grupos internos y externos. El implementar transacciones que no emplee papeles y actualice sus registros en tiempo real de los sistemas de contabilidad, requiere que los auditores externos a realizar una evaluación de tecnología de Información continua cuando la mayoría de la evidencia de auditoría existe en formato electrónico.

2. ECTIC consta de varias fases descritas en este artículo. Técnicas de auditoría continua presentada en este documento pueden ser utilizados para recoger la evidencia de auditoría al mismo tiempo como el procesamiento de la aplicación del sistema se proporciona a los auditores una alternativa viable a la utilización ex-post de auditoría y permitir que los auditores implementen una capacidad de prueba sorpresa.

3. El proceso de auditoría ha evolucionado a partir de la auditoría tradicional manual de un sistema de contabilidad con la documentación en papel, a los métodos de auditoría se utilizan actualmente en todo el equipo y la auditoría a través de computadoras, y con las nuevas tecnologías de información se está moviendo hacia el método de registros sin papel, electrónico, en línea, en tiempo real metodologías CA.
4. La nueva era de la mejora de TI permite a los auditores emplear ECTIC que actúa más como de prevención y disuasión, procedimientos contra errores de los estados financieros en lugar de un método correctivo de la eliminación de errores en los estados financieros que ya han ocurrido.

5. El uso de ETIC permite a los auditores establecer una serie de atributos predefinidos (por ejemplo, enfoque instantáneas, sistemas de control y registro) que continuamente podrá seleccionar, supervisar el sistema del cliente con la información contable y la estructura de control interno electrónica e informar de problemas a través de sistemas de alarma.

\section{REFERENCIAS BIBLIOGRÁFICAS}

1. Clark, R., Dillion, R. and Farrell, T. (1989), “'Continuous auditing", Internal Auditor, Spring, pp. 3-10.

2. Committee of Sponsoring Organizations of the Treadway Commission (COSO) (1992), Internal Control-integrated Framework, American Institute of Certified Public Accountants, September.

3. Intacct Corp. (2000), ' 'Web-based audit program is developed with Deloitte", Wall Street Journal, June 26, p. A13.

4. Pastor, C (2009), "El XBRL, nuevo lenguaje de comunicación de los Contadores Públicos del Perú”. Revista de Investigación Contable Quipukamayoc-UNMSM, 16(32), pp. 65-72.

5. Rezaee, Z., Ford, W.F. and Elam, R. (2000), " The role of internal auditors in a real-time accounting system", Internal Auditor, April, pp. 62-7.

6. Zarowin, S. and Harding, W.E. (2000), "Finally, business talks the same language", Journal of Accountancy, August, pp. 24-30. 